\title{
Molecular Profiling Predicts the Existence of Two Functionally Distinct Classes of Ovarian Cancer Stroma
}

\author{
Loukia N. Lili, ${ }^{1}$ Lilya V. Matyunina, ${ }^{1}$ L. DeEtte Walker, ${ }^{1}$ \\ Benedict B. Benigno, ${ }^{2}$ and John F. McDonald ${ }^{1,2}$ \\ ${ }^{1}$ Integrated Cancer Research Center, School of Biology and Parker H. Petit Institute of Bioengineering and Bioscience, \\ Georgia Institute of Technology, 315 Ferst Dr., Atlanta, GA 30332, USA \\ ${ }^{2}$ Ovarian Cancer Institute, 960 Johnson Ferry Road, Suite 130, Atlanta, GA 30342, USA
}

Correspondence should be addressed to John F. McDonald; mcgene@gatech.edu

Received 6 March 2013; Revised 16 April 2013; Accepted 18 April 2013

Academic Editor: Tao Huang

Copyright (C) 2013 Loukia N. Lili et al. This is an open access article distributed under the Creative Commons Attribution License, which permits unrestricted use, distribution, and reproduction in any medium, provided the original work is properly cited.

Although stromal cell signaling has been shown to play a significant role in the progression of many cancers, relatively little is known about its importance in modulating ovarian cancer development. The purpose of this study was to investigate the process of stroma activation in human ovarian cancer by molecular analysis of matched sets of cancer and surrounding stroma tissues. RNA microarray profiling of 45 tissue samples was carried out using the Affymetrix (U133 Plus 2.0) gene expression platform. Laser capture microdissection (LCM) was employed to isolate cancer cells from the tumors of ovarian cancer patients (Cepi) and matched sets of surrounding cancer stroma (CS). For controls, ovarian surface epithelial cells (OSE) were isolated from the normal (noncancerous) ovaries and normal stroma (NS). Hierarchical clustering of the microarray data resulted in clear separations between the OSE, Cepi, NS, and CS samples. Expression patterns of genes encoding signaling molecules and compatible receptors in the CS and Cepi samples indicate the existence of two subgroups of cancer stroma (CS) with different propensities to support tumor growth. Our results indicate that functionally significant variability exists among ovarian cancer patients in the ability of the microenvironment to modulate cancer development.

\section{Introduction}

The epithelial cells of the ovary interact with the cells of the surrounding microenvironment in order to regulate tissue homeostasis. Morphologically, the normal ovarian epithelial cells form a flat-to-cuboidal monolayer supported by a basement membrane. Cells located below this basement membrane are composed of various cell types collectively referred to as stromal cells. The most common types of stromal cells are fibroblasts, pericytes, endothelial cells, and various immune and inflammatory cells. Stromal and epithelial cells communicate through the secretion and binding of growth factors and other signaling molecules that promote reciprocal cellular responses appropriate for coordinated cell functions, for example, those required for the replication of ovarian surface epithelial cells following ovulation [1-3].

During cancer progression, genetic and epigenetic alterations lead to changes in the morphology and behavior of both epithelial and stromal cells by disrupting the tissue architecture and by interfering with signaling mechanisms. For example, signaling changes in a wide variety of developing cancer cells have been shown to result in the disruption of tissue homeostasis by inducing extracellular matrix (ECM) turnover, basement membrane disassociation, and increased stromal cell proliferation $[1,4]$.

Despite the well-documented role of stromal cell signaling in cancer progression, relatively few studies have been focused specifically on epithelial ovarian cancer-stromal interactions (EOC-SI). Previously reported studies on EOCSI have focused on particular stromal components $[5,6]$, on specific cell lines [7], and/or employed in-house fabricated microarrays of limited scope [8]. We report here the results of a study of EOC-SI using high-throughput gene expression (microarray) analysis of normal ovarian surface epithelial cells and cells captured from normal stroma, cancer epithelia, and cancer stroma using laser capture microdissection 
TABle 1: Patient samples used in this study.

\begin{tabular}{|c|c|c|c|c|c|}
\hline Patient ID & Age at time of surgery & Tissue for microarray & Histopathology & Stage & Grade \\
\hline 460 & 65 & OSE & WNL (within normal limits) & N/A & $\mathrm{N} / \mathrm{A}$ \\
\hline 552 & 41 & OSE & WNL & N/A & $\mathrm{N} / \mathrm{A}$ \\
\hline 563 & 66 & OSE & WNL & N/A & $\mathrm{N} / \mathrm{A}$ \\
\hline 567 & 78 & OSE & WNL & N/A & $\mathrm{N} / \mathrm{A}$ \\
\hline 434 & 41 & OSE/NS & WNL & N/A & N/A \\
\hline 437 & 54 & OSE/NS & WNL & N/A & $\mathrm{N} / \mathrm{A}$ \\
\hline 440 & 50 & OSE/NS & WNL & N/A & $\mathrm{N} / \mathrm{A}$ \\
\hline 448 & 63 & OSE/NS & WNL & N/A & $\mathrm{N} / \mathrm{A}$ \\
\hline 452 & 51 & OSE/NS & WNL & N/A & $\mathrm{N} / \mathrm{A}$ \\
\hline 463 & 48 & OSE/NS & WNL & N/A & $\mathrm{N} / \mathrm{A}$ \\
\hline 470 & 44 & OSE/NS & WNL & N/A & N/A \\
\hline 475 & 63 & OSE/NS & WNL & N/A & N/A \\
\hline 317 & 59 & Cepi & Serous adenocarcinoma & Ic & 3 \\
\hline 489 & 48 & Cepi & Serous adenocarcinoma & IV & 3 \\
\hline 528 & 66 & Cepi & Serous adenocarcinoma & IIIc & 3 \\
\hline 537 & 64 & Cepi & Serous adenocarcinoma & IIIa & 2 \\
\hline 542 & 61 & Cepi & Serous adenocarcinoma & IV & 3 \\
\hline 551 & 59 & Cepi & Serous adenocarcinoma & IIIc/IV & 3 \\
\hline 588 & 71 & Cepi & Serous adenocarcinoma & IIIc & 2 \\
\hline 606 & 54 & Cepi & Serous adenocarcinoma & IIIa & 3 \\
\hline 617 & 64 & Cepi & Serous adenocarcinoma & IIIc & 2 \\
\hline 620 & 62 & Cepi & Serous adenocarcinoma & III/IV & 3 \\
\hline 651 & 46 & Cepi & Serous adenocarcinoma & IIIb/IIIc & 3 \\
\hline 183 & 66 & Cepi/CS & Serous adenocarcinoma & III & 2 \\
\hline 369 & 52 & Cepi/CS & Serous adenocarcinoma & IIIC & 2 \\
\hline 229 & 58 & Cepi/CS & Serous adenocarcinoma & IIIC & 3 \\
\hline 242 & 63 & Cepi/CS & Serous adenocarcinoma & IIIb & 3 \\
\hline 336 & 63 & Cepi/CS & Serous adenocarcinoma & Ic & 3 \\
\hline 367 & 56 & Cepi/CS & Serous adenocarcinoma & II & 3 \\
\hline 413 & 49 & Cepi/CS & Serous adenocarcinoma & IIb & 3 \\
\hline
\end{tabular}

(LCM). Our results reveal the existence of two categories of ovarian cancer stroma. Analysis of ligand-receptor patterns of gene expression indicates that one of these classes of cancer stroma may be more permissive and one more resistant to associated cancer cell growth.

\section{Materials and Methods}

2.1. Tissue Collection. Tissues were collected at Northside Hospital (Atlanta, GA, USA) under appropriate Institutional Review Board protocols. Following resection, the tumor tissues were placed in cryotubes and immediately ( $<1$ minute) frozen in liquid nitrogen. Samples were transported on dry ice to Georgia Institute of Technology (Atlanta, GA, USA) and stored at $-80^{\circ} \mathrm{C}$. All tissues were examined, and diagnoses were made by a pathologist. The histopathology for each sample is listed in Table 1.

For each of the cancer tissue samples, $7 \mathrm{~mm}$ frozen sections were cut from samples embedded in cryomatrix (Shandon) and attached to uncharged microscope slides. Immediately following dehydration and staining (HistoGene,
LCM Frozen Section Staining Kit, Arcturus), slides were processed in an Autopix (Arcturus) instrument for laser capture microdissection (LCM) of cancer epithelial cells (CEPI), cancer stroma (CS), and normal stroma (NS) using CapSure Macro-LCM Caps (Arcturus). Approximately 30,000 cells were collected from each of the samples. Normal ovarian surface epithelial (OSE) cells were also collected from normal ovaries at the time of surgery by light brushing using a Cytobrush Plus (Medscand), immediately stabilized in RNAlater (Ambion), and subsequently stored at $-20^{\circ} \mathrm{C}$. Microscopic examination of all collected cells was carried out to confirm the integrity and purity of the samples.

2.2. RNA Extraction and Amplification. PicoPure RNA Isolation Kit (Arcturus) protocols were followed for RNA extraction from the LCM cells on the Macro-LCM caps in $25 \mu \mathrm{L}$ of extraction buffer. Normal OSE cells were pelleted from RNAlater; RNA was isolated with Trizol (Invitrogen) and purified with the PicoPure RNA Isolation Kit. RNA quality was verified for all samples on the Bioanalyzer RNA Pico Chip (Agilent Technologies). 
Total RNA from the above extractions was processed using the RiboAmp HS Kit (Arcturus) in conjunction with the IVT Labeling Kit from Affymetrix, to produce an amplified, biotin-labeled mRNA suitable for hybridizing to GeneChip Human Genome U133 Plus 2.0 Arrays (Affymetrix) following manufacturer's recommendations.

2.3. Microarray Data Analysis. We generated 45 individual gene expression profiles from 12 OSE brushings and $18 \mathrm{Cepi}$, $8 \mathrm{NS}$, and $7 \mathrm{CS}$ patient samples isolated by laser capture microdissection (LCM). Affymetrix CEL files were processed using the Affymetrix Expression Console (EC) Software Version 1.1 with the default MAS5.0 probeset normalization algorithm. The expression values from the 12 OSE, 18 Cepi, 8 NS, and 7 CS samples were $\log _{2}$ transformed and then averaged for each probeset across each sample type. The microarray data were deposited in the Gene Expression Omnibus (GSE38666).

Probesets (genes) with nearly constant expression values ( $\log _{2}$ normalized) across samples $(\mathrm{SD}<1)$ were excluded from further consideration. Of the 54,675 probesets on the U133 Plus 2.0 chip, 42,698 were thus retained. A fourway ANOVA was subsequently employed to identify genes significantly differentially expressed $(P \leq 0.001)$ across the four sample groups (OSE, Cepi, NS, and CS). These 6,654 genes were employed in the initial clustering analysis.

A subsequent comparison among the CS samples $\left(\mathrm{CS}_{1}\right.$ and $\mathrm{CS}_{2}$ ) alone was performed using a similar approach. Specifically, genes with nearly identical expression values $(\mathrm{SD}<1)$ across $\mathrm{CS}_{1}$ and $\mathrm{CS}_{2}$ were discarded, and the remaining 38,972 genes were subjected to an unpaired $t$-test to identify those genes that were significantly differentially expressed between the $\mathrm{CS}_{1}$ and $\mathrm{CS}_{2}$ subgroups $(P \leq 0.001$, 88 genes).

All heat maps were generated using the UPGMA (unweighted average) clustering method and the Euclidean distance similarity measure.

2.4. Ligand-Receptor Compatibility Analysis. For the ligandreceptor compatibility analysis, probesets associated with no or marginal expression across all 45 samples were discarded resulting in 5,865 differentially expressed genes. The presence or absence of the expression in samples was determined using the Affymetrix default MAS 5.0 decision algorithm. The MAS 5.0 algorithm uses Tukey's biweight estimator to provide a robust mean signal value and the Wilcoxon's rank test to calculate the significance of the signal or $P$ value and detection call (present, marginal, or absent) for each probeset. The $P$ values upon which the presence-absence calls for each ligand and receptor are based are presented in the appropriate Tables 1-5.

\section{Results}

3.1. Hierarchical Clustering Establishes Two Distinct Classes of Stroma among the Ovarian Cancer Patient Samples. Fortyfive gene expression profiles were generated from 12 OSE brushings and 18 Cepi, 8 NS, and 7 CS patient samples isolated by laser capture microdissection (LCM). The relevant histopathologies of these 45 samples are listed in Table 1. Expression analysis yielded 6,654 differentially expressed probesets among the four sample types (ANOVA, $P \leq 0.001$ ). Hierarchical clustering of these expression data resulted in clear separations between the OSE, Cepi, NS, and CS samples (Figure 1). Interestingly, the CS samples subdivided into two distinct groups. One $\left(\mathrm{CS}_{1}\right)$ was more closely associated with the NS samples, and the other $\left(\mathrm{CS}_{2}\right)$ was more closely associated with the Cepi samples.

One possibility is that the two subclasses of CS are simply a reflection of differential responses of stroma to molecular differences in the adjacent Cepi. If this were the case, we would expect to see a correlated substructure among the molecular profiles of the Cepi samples associated with the $\mathrm{CS}_{1}$ and $\mathrm{CS}_{2}$ subgroups. As shown in Figure 1, no such coordinated substructure pattern exists among the Cepi samples indicating that the two subclasses of CS are not merely a reflection of differential responses of the stroma to different Cepi subtypes.

As stated above, microscopic examination of LCM collected cells was carried out to validate the integrity of our samples. As a further confirmation, we conducted an additional computational analysis. In this analysis, probesets associated with no or marginal expression across all 45 samples were discarded resulting in 5,865 differentially expressed genes. If the reason for the presence of two classes of CS samples is that the $\mathrm{CS}_{2}$ class was a mixture of stroma and invasive Cepi cells, the gene expression levels in the putative mixed cancer stromal class $\left(\mathrm{CS}_{2}\right)$ would be expected to lie within the range of the maximum and minimum expression levels of the NS and Cepi groups (i.e., $\operatorname{avg}\left(\mathrm{CS}_{2}\right)<\mathrm{Min}$ and $\left.\operatorname{avg}\left(\mathrm{CS}_{2}\right)>\mathrm{Max}\right)$. Inconsistent with this prediction, we found that 2,342 or $40 \%(2,342 / 5,865)$ of the differentially expressed genes making up the $\mathrm{CS}_{2}$ class displayed values outside the predicted range of the mixed cell types. The fact that $60 \%$ of the expression values lie within the predicted range is not indicative of contamination but rather of the fact that not all genes are significantly overexpressed in the stroma relative to cancer samples. Collectively our microarray results are consistent with the microscopic examination in demonstrating the absence of infiltrating Cepi cells in the cancer stroma samples.

3.2. Gene Expression Patterns Are Consistent with the Existence of Ligand-Receptor Interactions between Cepi and CS. The significance of the presence of two distinct classes of ovarian cancer stroma may involve differential interaction between these stromal and the adjacent cancer cells. To explore this possibility, we first examined the expression levels of genes encoding signaling ligands and compatible receptors in the CS and Cepi datasets.

Two lists were established from the 5,865 differentially expressed probesets across the OSE, Cepi, NS, and CS samples. One list is comprised of all differentially expressed gene probes (note that each gene may be represented by multiple, nonoverlapping probes) encoding secreted ligands (ligand list) and the other of all expressed gene probes encoding 


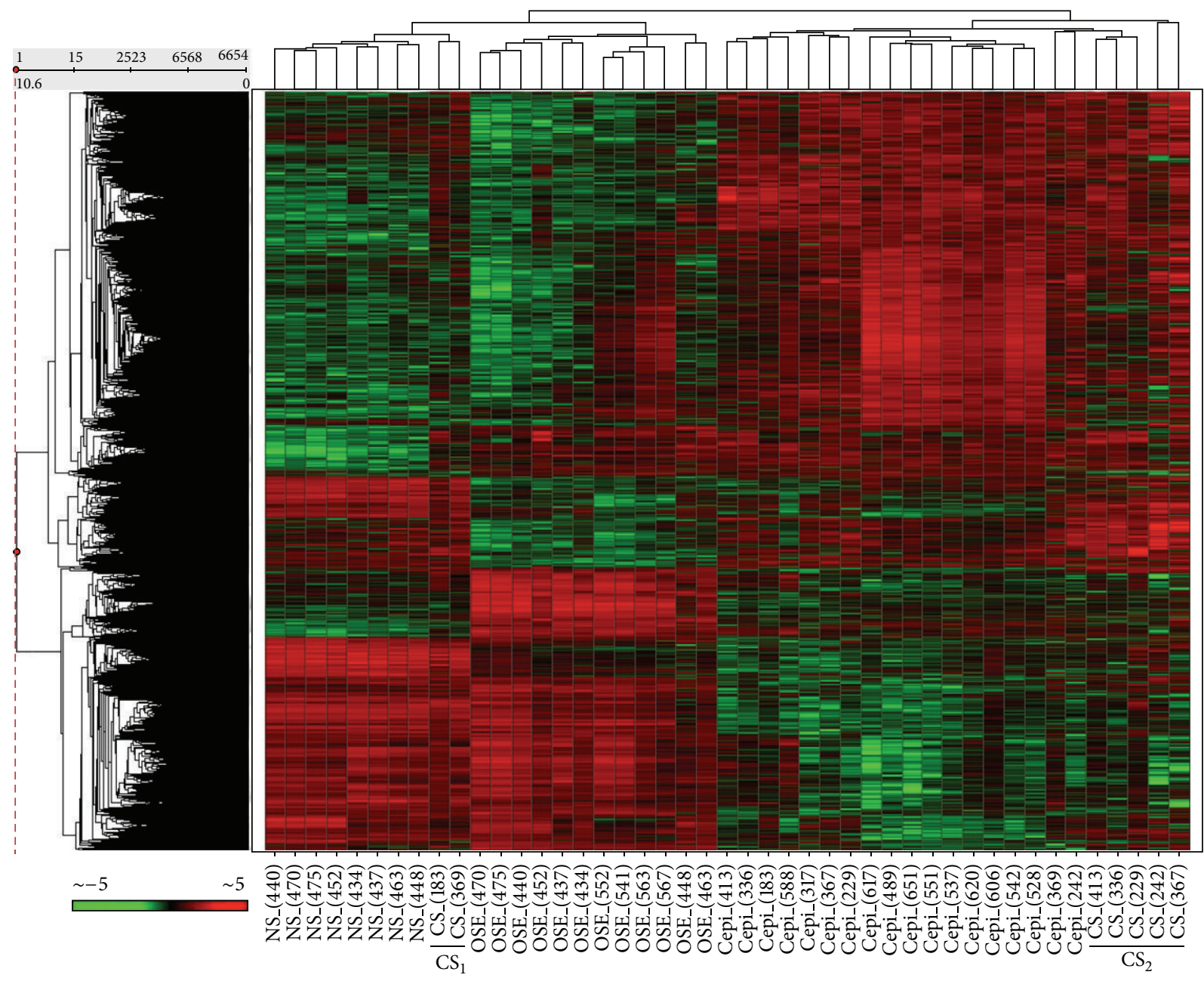

FIGURE 1: Hierarchical clustering of OSE, Cepi, NS, and CS expression profiles. The heat map was generated by $z$-score normalization of log 2 expression values from Affymetrix HG U133 Plus 2.0. The results show that the OSE, Cepi, NS, and CS samples cluster into separate groups. The CS samples clustered into two distinct subgroups $\left(\mathrm{CS}_{1}\right.$ and $\left.\mathrm{CS}_{2}\right)$.

surface receptors (receptor list) with documented binding affinity to the differentially expressed ligands (compatible ligands and receptors). The ligand list consists of 34 CS and 36 Cepi ligands while the receptor list is comprised of 20 Cepi and 21 CS receptors (Tables $2(\mathrm{a})$ and $2(\mathrm{~b})$ ).

We considered the expression of a ligand in CS (or Cepi) and its compatible receptor in Cepi (or CS) to be indicative of a potential CS-Cepi signaling interaction. Based on these criteria, we identified potential epithelial cancer-stroma signaling interactions (34 CS ligands and 20 Cepi receptors, see Table 2(a) and 36 Cepi ligands and 21 CS receptors, see Table 2(b)). Of these, there were 17 compatible pairs for both the CS ligands-Cepi receptors and Cepi ligands-CS receptors interactions (Table 3). Viewed from the perspective of individual genes (i.e., combining multiple probes of the same genes), there were 12 unique CS ligand-Cepi receptor pairs and 12 unique Cepi ligand-CS receptor pairs in our observed dataset (Table 4).

To determine if the observed coexpression of these 17 pairs of compatible ligands and receptors (probes) was greater than what would be expected by chance, we generated two lists. One list of observed data consisted of the expressed probes of the 17 CS ligands and 17 compatible Cepi receptors (Table 3(a)) and the other of the 17 expressed Cepi ligands and 17 CS receptors (Table 3(b)). A second list of random associations was generated using the same number of pairings as in the observed list (17 random pairs) and randomly selecting 17 pairs of ligands and receptors. One randomly selected CS (or Cepi) ligand from the pool of the 34 CS-(or 36 Cepi-) expressed ligands (Table 2(a)) was paired with one randomly selected Cepi (or CS) receptor from the pool of the 20 Cepi-(or 21 CS-) expressed receptors (Table 2(b)). These random associations were generated 100 times, and each time the number of biologically compatible ligand-receptor pairs arising by chance was counted. The number of biologically compatible interactions in the observed data (17) was then compared to the number of compatible interactions scored from the randomized associations using $z$-statistics. Two types of comparisons were performed, one for the pairs of CS ligands and Cepi receptors and another for the pairs of Cepi 
TABLE 2: The 34 CS-expressed ligands with the 20 expressed Cepi receptors (a) and the 36 Cepi-expressed ligands with the 21 expressed CS receptors (b).

(a)

\begin{tabular}{|c|c|c|c|}
\hline \multicolumn{2}{|c|}{ CS ligands } & \multicolumn{2}{|c|}{ Cepi receptors } \\
\hline Gene symbol & Probeset ID & Gene symbol & Probeset ID \\
\hline${ }^{* * *}$ CXCL1 & 204470_at & ${ }^{* * * *}$ CXCR 4 & 217028_at \\
\hline${ }^{*}$ CXCL3 & 207850_at & ${ }^{* *}$ FGFR2 & 208228_s_at \\
\hline *** CXCL9 & 203915_at & ${ }^{* * *}$ FGFR3 & 204379_s_at \\
\hline *** CXCL10 & 204533_at & ${ }^{* *} \mathrm{MET}$ & 203510_at \\
\hline${ }^{* * *}$ CXCL11 & 210163_at & ${ }^{*}$ TGFBR2 & 207334_s_at \\
\hline *** CXCL12 & 209687_at & ${ }^{* * *}$ TGFBR2 & 208944_at \\
\hline${ }^{* * *}$ CXCL12 & 203666_at & ${ }^{* *}$ TGFBR3 & 204731_at \\
\hline *** CXCL13 & 205242_at & ${ }^{* * *}$ TGFBR3 & 226625_at \\
\hline${ }^{* *}$ CXCL16 & 223454_at & *** PDGFRA & 203131_at \\
\hline CXCL17 & 226960_at & *PDGFRA & 1554828_at \\
\hline${ }^{*} \mathrm{FGF} 1$ & 205117_at & ${ }^{* * *}$ IL1R1 & 202948_at \\
\hline *FGF2 & 204422_s_at & *IL1R1 & 215561_s_at \\
\hline${ }^{* * *}$ FGF7 & 1554741_s_at & ${ }^{*}$ IL1R2 & 205403_at \\
\hline${ }^{*}$ FGF9 & 239178_at & ${ }^{* *}$ IL7R & 226218_at \\
\hline${ }^{* *}$ FGF9 & 206404_at & ${ }^{* *}$ IL10RA & 204912_at \\
\hline ***FGF13 & 205110_s_at & ${ }^{* *}$ FZD1 & 204451_at \\
\hline${ }^{*} \mathrm{HGF}$ & 210997_at & ${ }^{* *}$ FZD2 & 210220_at \\
\hline${ }^{* * *}$ IGF1 & 209540_at & ${ }^{* *}$ FZD7 & 203705_s_at \\
\hline *IGF2 & 202409_at & ${ }^{* * *}$ FZD7 & 203706_s_at \\
\hline${ }^{*}$ TGFA & 205016_at & ${ }^{* *}$ FZD10 & 219764_at \\
\hline${ }^{* * *}$ TGFB2 & 209909_s_at & & \\
\hline${ }^{*}$ PDGFA & 205463_s_at & & \\
\hline ***PDGFD & 219304_s_at & & \\
\hline * IL7 & 206693_at & & \\
\hline **** IL15 & 205992_s_at & & \\
\hline ** IL16 & 209828_s_at & & \\
\hline${ }^{* *}$ IL17D & 227401_at & & \\
\hline${ }^{* *}$ IL18 & 206295_at & & \\
\hline${ }^{* *}$ WNT2B & 206458_s_at & & \\
\hline${ }^{*}$ WNT7A & 210248_at & & \\
\hline${ }^{* * *}$ WNT5A & 213425_at & & \\
\hline ****VEGFA & 210512_s_at & & \\
\hline${ }^{*}$ VEGFA & 210513_s_at & & \\
\hline${ }^{*}$ VEGFA & 211527_x_at & & \\
\hline
\end{tabular}

(b)

\begin{tabular}{lccc}
\hline \multicolumn{2}{c}{ Cepi ligands } & \multicolumn{2}{c}{ CS receptors } \\
Gene symbol & Probeset ID & Gene symbol & Probeset ID \\
\hline${ }^{* * *}$ CXCL1 & 204470_at & ${ }^{* * *}$ CXCR4 & 217028_at \\
${ }^{* *}$ CXCL3 & 207850_at & ${ }^{* *}$ FGFR2 & 208228_s_at \\
${ }^{* * *}$ CXCL9 & 203915_at & ${ }^{*}$ FGFR3 & 204379_s_at \\
${ }^{* * *}$ CXCL10 & 204533_at & IL12RB1 & 1552584_at \\
${ }^{* * *}$ CXCL11 & 210163_at & ${ }^{* * *}$ IL1R1 & 202948_at \\
${ }^{*}$ CXCL12 & 203666_at & ${ }^{* * *}$ TGFBR2 & 208944_at \\
${ }^{*}$ CXCL12 & 209687_at & ${ }^{* * *}$ TGFBR3 & 204731_at \\
${ }^{* *}$ CXCL13 & 205242_at & ${ }^{* * *}$ TGFBR3 & 226625_at \\
${ }^{* *}$ CXCL16 & 223454_at & ${ }^{* * *}$ PDGFRA & 203131_at \\
\hline
\end{tabular}

(b) Continued.

\begin{tabular}{|c|c|c|c|}
\hline \multicolumn{2}{|c|}{ Cepi ligands } & \multicolumn{2}{|c|}{ CS receptors } \\
\hline Gene symbol & Probeset ID & Gene symbol & Probeset ID \\
\hline CXCL17 & 226960_at & ${ }^{* *}$ PDGFRA & 215305_at \\
\hline${ }^{*} \mathrm{FGF} 1$ & 205117_at & ${ }^{* * *} \mathrm{MET}$ & 203510_at \\
\hline${ }^{* * *}$ FGF9 & 206404_at & ${ }^{* *}$ IL1R1 & 215561_s_at \\
\hline${ }^{* * *}$ FGF9 & 239178_at & *IL1R2 & 205403_at \\
\hline${ }^{*}$ FGF11 & 227271_at & ${ }^{* *}$ IL7R & 226218_at \\
\hline${ }^{* * *}$ FGF18 & 231382_at & ${ }^{* *}$ IL10RA & 204912_at \\
\hline${ }^{*}$ FGF18 & 211029_x_at & ${ }^{*}$ IL21R & 221658_s_at \\
\hline${ }^{*}$ FGF18 & 206987_x_at & ${ }^{* *}$ FZD1 & 204451_at \\
\hline${ }^{* *} \mathrm{FGF} 18$ & 214284_s_at & ${ }^{* *}$ FZD2 & 210220_at \\
\hline${ }^{* *}$ TGFA & 205016_at & ${ }^{* * *}$ FZD7 & 203705_s_at \\
\hline${ }^{* *}$ TGFB2 & 209909_s_at & ${ }^{* *}$ FZD7 & 203706_s_at \\
\hline **PDGFA & 205463_s_at & ${ }^{* * * *}$ FZD10 & 219764_at \\
\hline *** PDGFD & 219304_s_at & & \\
\hline *** IGF1 & 209540_at & & \\
\hline *IL7 & 206693_at & & \\
\hline${ }^{*} \mathrm{IL1B}$ & 39402_at & & \\
\hline *** IL15 & 205992_s_at & & \\
\hline${ }^{* *}$ IL18 & 206295_at & & \\
\hline${ }^{* *} \mathrm{WNT} 2$ & 205648_at & & \\
\hline${ }^{* *} \mathrm{WNT} 2 \mathrm{~B}$ & 206458_s_at & & \\
\hline ***WNT5A & 213425_at & & \\
\hline *WNT7A & 210248_at & & \\
\hline "**WNT11 & 206737_at & & \\
\hline${ }^{* * *}$ VEGFA & 210512_s_at & & \\
\hline${ }^{* * *}$ VEGFA & 210513_s_at & & \\
\hline *VEGFA & 211527_x_at & & \\
\hline${ }^{* *}$ VEGFA & 212171_x_at & & \\
\hline
\end{tabular}

Significance of detection calls: ${ }^{*} P \leq 0.05,{ }^{* *} P \leq 0.005$, and ${ }^{* * *} P \leq 0.0005$.

ligands and CS receptors. For both comparisons, the observed number of biologically compatible ligand-receptor pairs was significantly greater than what is expected by chance (CS ligands-Cepi receptors $z$-score $=-4.68, P \leq 0.0002$; Cepi ligands-CS receptors $z$-score $=-4.35, P \leq 0.0002$ ). Thus, the observed coexpression of pairs of compatible ligands and receptors is biologically significant.

3.3. Specific Ligand-Receptor Pairs between Cepi and CS Show Differential Gene Expression in the Two CS Classes. Of the 24 compatible pairs of ligand- and receptor-encoding genes listed in Table 4, most display similar expression patterns between $\mathrm{CS}_{1}$ and $\mathrm{CS}_{2}$. However, 6 of the ligand and receptor pairs display differential patterns of expression between the two groups of $\mathrm{CS}$ suggesting that $\mathrm{CS}_{2}$ may be a more conducive microenvironment for tumor growth (Table 5). For example, the FGF2 ligand, a documented inhibitor of tumor growth [9], is expressed in NS and in $\mathrm{CS}_{1}$ but not in $\mathrm{CS}_{2}$. Since a compatible receptor of this inhibitor (FGFR3) is expressed in Cepi, $\mathrm{CS}_{2}$ may be a more conducive microenvironment for tumor growth than $\mathrm{CS}_{1}$. The interleukin-7 (IL7) ligand has been previously implicated as an inducer of tumor growth 
TABLE 3: The expressed, compatible ligands and receptors as potential interactions between the Cepi and the CS samples from Tables 2(a) and 2(b).

(a)

\begin{tabular}{lccc}
\hline CS ligands & Probesets & $\begin{array}{c}\text { Compatible } \\
\text { Cepi receptors }\end{array}$ & Probesets \\
\hline${ }^{* * *}$ CXCL12 & 203666_at & ${ }^{* * *}$ CXCR4 & 217028_at \\
${ }^{* * *}$ CXCL12 & 209687_at & ${ }^{* * *}$ CXCR4 & 217028_at \\
${ }^{*}$ FGF1 & 205117_at & ${ }^{* *}$ FGFR2 & 208228_s_at \\
${ }^{*}$ FGF1 & 205117_at & ${ }^{*}$ FGFR3 & 204379_s_at \\
${ }^{*}$ FGF2 & 204422_s_at & ${ }^{*}$ FGFR3 & 204379_s_at \\
${ }^{* *}$ FGF9 & 206404_at & ${ }^{*}$ FGFR3 & 204379_s_at \\
${ }^{*}$ FGF9 & 239178_at & ${ }^{*}$ FGFR3 & 204379_s_at \\
${ }^{*}$ HGF & 210997_at & ${ }^{*}$ MET & 203510_at \\
${ }^{*}$ PDGFA & 205463_s_at & ${ }^{*}$ PDGFRA & 1554828_at \\
${ }^{*}$ PDGFA & 205463_s_at & ${ }^{* * *}$ PDGFRA & 203131_at \\
${ }^{* * *}$ TGFB2 & 209909_s_at & ${ }^{*}$ TGFBR2 & 207334_s_at \\
${ }^{* * *}$ TGFB2 & 209909_s_at & ${ }^{* * *}$ TGFBR2 & 208944_at \\
${ }^{*}$ WNT2 & 205648_at & ${ }^{*}$ FZD2 & 210220_at \\
${ }^{* *}$ WNT2B & 206458_s_at & ${ }^{*}$ FZD10 & 219764_at \\
${ }^{*}$ WNT7A & 210248_at & ${ }^{*}$ FZD7 & 203705_s_at \\
${ }^{*}$ WNT7A & 210248_at & ${ }^{* *}$ FZD7 & 203706_s_at \\
${ }^{*}$ IL7 & 206693_at & ${ }^{* *}$ IL7R & 226218_at \\
\hline
\end{tabular}

(b)

\begin{tabular}{lccc}
\hline Cepi ligands & Probesets & $\begin{array}{c}\text { Compatible CS } \\
\text { receptors }\end{array}$ & Probesets \\
\hline${ }^{*}$ CXCL12 & 203666_at & ${ }^{* * *}$ CXCR4 & 217028_at \\
${ }^{*}$ CXCL12 & 209687_at & ${ }^{* * *}$ CXCR4 & 217028_at \\
${ }^{*}$ FGF1 & 205117_at & ${ }^{* *}$ FGFR2 & 208228_s_at \\
${ }^{*}$ FGF1 & 205117_at & ${ }^{*}$ FGFR3 & 204379_s_at \\
${ }^{*}$ FGF9 & 206404_at & ${ }^{*}$ FGFR3 & 204379_s_at \\
FGF9 & 239178_at & ${ }^{*}$ FGFR3 & 204379_s_at \\
${ }^{*}$ PDGFA & 205463_s_at & ${ }^{* * *}$ PDGFRA & 203131_at \\
${ }^{*}$ PDGFA & 205463_s_at & ${ }^{*}$ PDGFRA & 215305_at \\
${ }^{*}$ TGFB2 & 209909_s_at & ${ }^{* * *}$ TGFBR2 & 208944_at \\
${ }^{*}$ WNT2 & 205648_at & ${ }^{*}$ FZD2 & 210220_at \\
${ }^{*}$ WNT2B & 206458_s_at & ${ }^{* * *}$ FZD10 & 219764_at \\
${ }^{*}$ WNT7A & 210248_at & ${ }^{* * *}$ FZD7 & 203705_s_at \\
${ }^{*}$ WNT7A & 210248_at & ${ }^{* *}$ FZD7 & 203706_s_at \\
${ }^{*}$ IL1B & 39402_at & ${ }^{* * *}$ IL1R1 & 202948_at \\
${ }^{*}$ IL1B & 39402_at & ${ }^{*}$ IL1R2 & 205403_at \\
${ }^{*}$ IL1B & 39402_at & ${ }^{*}$ IL1R1 & 215561_s_at \\
${ }^{*}$ IL7 & 206693_at & ${ }^{*}$ IL7R & 226218_at \\
\hline
\end{tabular}

Significance of detection calls: ${ }^{*} P \leq 0.05,{ }^{* *} P \leq 0.005$, and ${ }^{* * *} P \leq 0.0005$.

in lymphoblastic leukemia [10], prostate cancer [11], breast cancer [12], and colorectal cancer [13]. IL7 is expressed in $\mathrm{CS}_{2}$ but not in $\mathrm{CS}_{1}$, again suggesting that $\mathrm{CS}_{2}$ may be a more conducive microenvironment for tumor growth than $\mathrm{CS}_{1}$.
TABLE 4: The unique compatible ligands and receptors as potential interactions between the Cepi and the CS samples when multiple probes from Tables 3(a) and 3(b) are combined.

\begin{tabular}{|c|c|c|c|}
\hline CS ligands & $\begin{array}{c}\text { Compatible } \\
\text { Cepi receptors }\end{array}$ & Cepi ligands & $\begin{array}{c}\text { Compatible CS } \\
\text { receptors }\end{array}$ \\
\hline *** CXCL12 & ${ }^{* * *}$ CXCR4 & ${ }^{*} \mathrm{CXCL} 12$ & *** CXCR4 \\
\hline${ }^{*} \mathrm{FGF} 1$ & ${ }^{* *}$ FGFR2 & ${ }^{*} \mathrm{FGF} 1$ & ${ }^{* *}$ FGFR2 \\
\hline${ }^{*} \mathrm{FGF} 1$ & ${ }^{*}$ FGFR3 & ${ }^{*}$ FGF1 & ${ }^{*}$ FGFR3 \\
\hline${ }^{*}$ FGF2 & ${ }^{*}$ FGFR3 & ${ }^{* *}$ FGF9 & ${ }^{*}$ FGFR3 \\
\hline${ }^{* *}$ FGF9 & ${ }^{*}$ FGFR3 & ${ }^{* *}$ PDGFA & ${ }^{* * *}$ PDGFRA \\
\hline${ }^{*} \mathrm{HGF}$ & ${ }^{*} \mathrm{MET}$ & ${ }^{* *}$ TGFB2 & ${ }^{* * *}$ TGFBR2 \\
\hline${ }^{*}$ PDGFA & ${ }^{* * *}$ PDGFRA & ${ }^{* *}$ IL7 & ${ }^{*}$ IL7R \\
\hline${ }^{* * *}$ TGFB2 & ${ }^{* * *}$ TGFBR2 & ${ }^{* *}$ IL1B & ${ }^{*}$ IL1R1 \\
\hline${ }^{*}$ IL7 & ${ }^{* *}$ IL7R & ${ }^{* *}$ IL1B & ${ }^{*}$ IL1R2 \\
\hline${ }^{*}$ WNT2 & ${ }^{*}$ FZD2 & ${ }^{* *}$ WNT2 & ${ }^{*} \mathrm{FZD} 2$ \\
\hline${ }^{* *}$ WNT2B & ${ }^{*}$ FZD10 & ${ }^{* *} \mathrm{WNT} 2 \mathrm{~B}$ & ${ }^{* * *}$ FZD10 \\
\hline${ }^{*}$ WNT7A & ${ }^{* *}$ FZD7 & ${ }^{* *}$ WNT7A & ${ }^{* * *}$ FZD7 \\
\hline
\end{tabular}

Significance of detection calls: ${ }^{*} P \leq 0.05,{ }^{* *} P \leq 0.005$, and ${ }^{* * *} P \leq 0.0005$.

The well-documented cancer-inducing ligands FGF1 and FGF9 [14-16] are both highly expressed in Cepi. The fact that the compatible FGFR3 receptor is expressed in $\mathrm{CS}_{2}$ but not in $\mathrm{CS}_{1}$ again suggests that $\mathrm{CS}_{2}$ is a more favorable microenvironment for ovarian cancer growth than $\mathrm{CS}_{1}$.

The WNT family of genes is involved in a variety of developmental processes, and aberrant expression of various members of WNT genes has been implicated in cancer [17]. For example, WNT7A is a ligand present in the extracellular matrix that participates in the sexual development of the Mullerian ducts [18]. Recent in vivo mouse studies suggest that WNT7A is an inducer of ovarian cancer growth [19]. Consistent with this interpretation, WNT7A has recently been identified as a potential early stage biomarker of human ovarian cancer [20]. The fact that WNT7A is expressed in $\mathrm{CS}_{2}$ but not in $\mathrm{CS}_{1}$ is also consistent with the hypothesis that $\mathrm{CS}_{2}$ may be a more conducive microenvironment for ovarian cancer growth than $\mathrm{CS}_{1}$.

A second member of the WNT family, WNT2B, is expressed in $\mathrm{CS}_{1}$ but not $\mathrm{CS}_{2}$ suggesting, contrary to what is presented above, that $\mathrm{CS}_{1}$ may be more permissive for cancer growth. However, the fact that WNT2B has been previously reported to be expressed in normal ovaries [21] coupled with our finding that it is also expressed in NS makes interpreting the significance of the dichotomy in WNT2B expression between $\mathrm{CS}_{1}$ and $\mathrm{CS}_{2}$ ambiguous.

\section{Discussion}

Cancer progression is a dynamic process involving cellular adaptation and survival that is, in part, driven by signaling interactions between participating cells. Many signaling interactions have been documented to take place between cancer epithelial cells and the surrounding stroma [22]. Early in tumor development, cancer cells produce growth factors that are believed to modulate or "activate" the surrounding 
TABLE 5: The unique compatible ligands and receptors from Table 4 showing the expression pattern in NS, $\mathrm{CS}_{1}, \mathrm{CS}_{2}$, and Cepi. The 6 bold signals had the same expression in NS and $\mathrm{CS}_{1}$ but different expression between $\mathrm{CS}_{1}$ and $\mathrm{CS}_{2}$ despite the fact that their compatible signals in Cepi were always expressed.

\begin{tabular}{|c|c|c|c|c|c|}
\hline NS & Ligands & $\mathrm{CS}_{1}$ & $\mathrm{CS}_{2}$ & Receptors & Cepi \\
\hline+ & CXCL12 & + & + & CXCR4 & + \\
\hline- & FGF1 & + & + & FGFR2 & - \\
\hline- & FGF1 & + & + & FGFR3 & + \\
\hline+ & FGF2 & + & - & FGFR3 & + \\
\hline+ & FGF9 & + & + & FGFR3 & + \\
\hline- & HGF & + & + & MET & + \\
\hline+ & PDGFA & + & + & PDGFRA & + \\
\hline+ & TGFB2 & + & + & TGFBR2 & + \\
\hline- & IL7 & - & + & IL7R & + \\
\hline- & WNT2 & + & + & FZD2 & + \\
\hline+ & WNT2B & + & - & FZD2 & + \\
\hline- & WNT7A & - & + & FZD7 & + \\
\hline NS & Receptors & $\mathrm{CS}_{1}$ & $\mathrm{CS}_{2}$ & Ligands & Cepi \\
\hline+ & CXCR4 & + & + & CXCL12 & + \\
\hline+ & FGFR2 & + & + & FGF1 & + \\
\hline- & FGFR3 & - & + & FGF1 & + \\
\hline- & FGFR3 & - & + & FGF9 & + \\
\hline+ & PDGFRA & + & + & PDGFA & + \\
\hline+ & TGBFR2 & + & + & TGFB2 & + \\
\hline+ & IL1R1 & + & + & IL1B & + \\
\hline- & IL1R2 & + & - & IL1B & + \\
\hline+ & IL7R & + & + & IL7 & + \\
\hline+ & FZD2 & + & + & WNT2 & + \\
\hline+ & FZD2 & + & + & WNT2B & + \\
\hline+ & FZD7 & + & + & WNT7A & + \\
\hline
\end{tabular}

Expression is denoted with "+" (i.e., there is at least one Affymetrix present call with detection $P$ value $\leq 0.05$ ) and nonexpression with "-" (i.e., there are no Affymetrix present calls in the samples with detection $P$ value $\leq 0.05$ ).

stroma in order to convert the stroma into a supportive microenvironment for cancer growth $[2,14]$. For example, it has been shown that growth factors secreted by macrophages can contribute to cancer progression and metastasis [23]. Other inflammatory cells such as lymphocytes, neutrophils, mast cells, T-regulatory cells, and platelets also have been shown to have the potential to support tumor progression by negatively regulating the anticancer host immune response [24-26]. Fibroblasts, the major component of the stroma, have been shown to be able to participate actively in the malignant progression of cancer by producing growth factors, various chemokines, and extra cellular matrix components that facilitate the production of endothelial cells and pericytes conducive to tumor growth [14, 27].

The purpose of this study was to investigate the process of stroma activation within the context of ovarian cancer. Toward this end, we conducted RNA microarray profiling of 45 tissue samples using the Affymetrix (U133 Plus2) gene expression platform. Laser capture microdissection (LCM) was used to isolate cancer cells from the tumors of 18 ovarian cancer patients (Cepi). For 7 of these patients, a matched set of surrounding cancer stroma (CS) was also collected. For controls, we isolated surface epithelial cells (OSE) from the normal (noncancerous) ovaries of 12 individuals including matched sets of samples of OSE and normal stroma (NS) from 8 of these patients.

Unsupervised hierarchical clustering of the microarray data resulted in the expected separation between the OSE and Cepi samples. Consistent with models of stromal activation, we also observed significant separation between the NS and CS samples. Somewhat unexpected, however, was our finding that the CS samples clustered into two distinct subgroups $\left(\mathrm{CS}_{1}\right.$ and $\left.\mathrm{CS}_{2}\right)$.

Based on patterns of coexpression of ligand and receptor encoding genes, we determined that 6 biologically compatible pairs of ligands and receptors are differentially expressed between Cepi and the $\mathrm{CS}_{1}$ and $\mathrm{CS}_{2}$ cancer stroma. The patterns of differential expression between the compatible ligands and receptors are consistent with the hypothesis that $\mathrm{CS}_{2}$ may be a more conducive microenvironment for tumor growth (Table 5). For example, the expression of tumor promoting ligands in Cepi is always matched with the expression of compatible receptors in $\mathrm{CS}_{2}$ but not in $\mathrm{CS}_{1}$.

The fact that certain tumor microenvironments are capable of inhibiting tumor growth and/or development is well established. For example, macrophages can act as anticancer agents within the context of the innate immune response [28]. Likewise, fibroblasts, in some cellular contexts, have been shown to revert tumor cells to a normal, noncancerous phenotype $[9,29]$. Normal ovarian stromal cells have been shown to significantly inhibit ovarian cancer cell growth when coinjected into nude mice [30].

The apparently innate anticancer properties of normal stroma are generally considered to be transient giving way to the "activation" of procancer growth signals induced by cancer cells as the tumors progress [1]. However, since the majority of the patients associated with the $\mathrm{CS}_{1}$ class of cancer stroma have, like the majority of the cancer patients included in our study, already progressed to advanced staged disease (Table 1), it is unlikely that the $\mathrm{CS}_{1}$ molecular profile represents a transient condition. Rather, our results suggest that variability exists among ovarian cancer patients with respect to the propensity of normal stroma to become activated.

\section{Conclusions}

An understanding of the potential clinical significance of the observed molecular dichotomy between ovarian cancer stroma is beyond the scope of this present study. However, it is relevant to note that all of the cancers associated with the putatively more permissive $\mathrm{CS}_{2}$ cancer stroma were classified as grade 3 while those associated with the putatively more resistant $\mathrm{CS}_{1}$ cancer stroma were classified as grade 2. The fact that no distinction was apparent between the molecular profiles of grade 2 and grade 3 Cepi samples (Figure 1) suggests that cancer grade may, at least in part, be determined by the relative permissiveness of the tumor 
microenvironment. Molecular profiling of larger numbers of matched sets of ovarian cancer and stroma samples will be required to adequately test this hypothesis. Nevertheless, the current results are consistent with the hypothesis that the microenvironment plays a significant role in ovarian cancer development and suggest that functionally significant variability may exist among ovarian cancer patients in the ability of the microenvironment to modulate cancer development.

\section{Conflict of Interests}

The authors declare that there is no conflict of interests.

\section{Acknowledgments}

The project was supported by grants from the Ovarian Cancer Institute (Atlanta), Northside Hospital (Atlanta), the Robinson Family Foundation, Ovarian Cycle Foundation, and the Deborah Willingham Endowment Fund. The authors also wish to thank Dr. Nathan J. Bowen for his advise in early stages of the project.

\section{References}

[1] L. A. Liotta and E. C. Kohn, "The microenvironment of the tumour-host interface," Nature, vol. 411, no. 6835, pp. 375-379, 2001.

[2] N. A. Bhowmick and H. L. Moses, "Tumor-stroma interactions," Current Opinion in Genetics and Development, vol. 15, no. 1, pp. 97-101, 2005.

[3] T. D. Tlsty and L. M. Coussens, "Tumor stroma and regulation of cancer development," Annual Review of Pathology, vol. 1, pp. 119-150, 2006.

[4] D. Hanahan and R. A. Weinberg, "The hallmarks of cancer," Cell, vol. 100, no. 1, pp. 57-70, 2000.

[5] A. A. Kamat, M. Fletcher, L. M. Gruman et al., "The clinical relevance of stromal matrix metalloproteinase expression in ovarian cancer," Clinical Cancer Research, vol. 12, no. 6, pp. 1707$1714,2006$.

[6] L. Zhang, N. Yang, J. W. Park et al., "Tumor-derived vascular endothelial growth factor up-regulates angiopoietin-2 in host endothelium and destabilizes host vasculature, supporting angiogenesis in ovarian cancer," Cancer Research, vol. 63, no. 12, pp. 3403-3412, 2003.

[7] C. Porcile, A. Bajetto, F. Barbieri et al., "Stromal cell-derived factor-1 $\alpha$ (SDF-1 $\alpha /$ CXCL12) stimulates ovarian cancer cell growth through the EGF receptor transactivation," Experimental Cell Research, vol. 308, no. 2, pp. 241-253, 2005.

[8] E. Wang, Y. Ngalame, M. C. Panelli et al., "Peritoneal and subperitoneal stroma may facilitate regional spread of ovarian cancer," Clinical Cancer Research, vol. 11, no. 1, pp. 113-122, 2005.

[9] P. Micke and A. Östman, "Tumour-stroma interaction: cancerassociated fibroblasts as novel targets in anti-cancer therapy?" Lung Cancer, vol. 45, supplement 2, pp. S163-S175, 2004.

[10] A. Silva, A. Gírio, I. Cebola, C. I. Santos, F. Antunes, and J. T. Barata, "Intracellular reactive oxygen species are essential for PI3K/Akt/mTOR-dependent IL-7-mediated viability of T-cell acute lymphoblastic leukemia cells," Leukemia, vol. 25, no. 6, pp. 960-967, 2011.
[11] C. Schroten, N. F. Dits, E. W. Steyerberg et al., "The additional value of TGF $\beta 1$ and IL-7 to predict the course of prostate cancer progression," Cancer Immunology, Immunotherapy, vol. 61, no. 6, pp. 905-910, 2012.

[12] M. A. A. Al-Rawi, R. E. Mansel, and W. G. Jiang, "Interleukin7 (IL-7) receptor (IL-7R) signalling complex in human solid tumours," Histology and Histopathology, vol. 18, no. 3, pp. 911923, 2003.

[13] M. J. Maeurer, W. Walter, D. Martin et al., "Interleukin-7 (IL-7) in colorectal cancer: IL-7 is produced by tissues from colorectal cancer and promotes preferential expansion of tumour infiltrating lymphocytes," Scandinavian Journal of Immunology, vol. 45, no. 2, pp. 182-192, 1997.

[14] N. A. Bhowmick, E. G. Neilson, and H. L. Moses, "Stromal fibroblasts in cancer initiation and progression," Nature, vol. 432, no. 7015, pp. 332-337, 2004.

[15] C. Jin, F. Wang, X. Wu, C. Yu, Y. Luo, and W. L. McKeehan, "Directionally specific paracrine communication mediated by epithelial FGF9 to stromal FGFR3 in two-compartment premalignant prostate tumors," Cancer Research, vol. 64, no. 13, pp. 4555-4562, 2004.

[16] N. Turner and R. Grose, "Fibroblast growth factor signalling: from development to cancer," Nature Reviews Cancer, vol. 10, no. 2, pp. 116-129, 2010.

[17] T. Reya and H. Clevers, "Wnt signalling in stem cells and cancer," Nature, vol. 434, no. 7035, pp. 843-850, 2005.

[18] T. D. Bui, M. Lako, S. Lejeune et al., "Isolation of a full-length human WNT7A gene implicated in limb development and cell transformation, and mapping to chromosome 3p25," Gene, vol. 189, no. 1, pp. 25-29, 1997.

[19] S. Yoshioka, M. L. King, S. Ran et al., "WNT7A regulates tumor growth and progression in ovarian cancer through the $\mathrm{WNT} / \beta$ catenin pathway," Molecular Cancer Research, vol. 10, no. 3, pp. 469-482, 2012.

[20] A. B. Tchagang, A. H. Tewfik, M. S. DeRycke, K. M. Skubitz, and A. P. N. Skubitz, "Early detection of ovarian cancer using group biomarkers," Molecular Cancer Therapeutics, vol. 7, no. 1, pp. 27-37, 2008.

[21] A. Ricken, P. Lochhead, M. Kontogiannea, and R. Farookhi, "Wnt signaling in the ovary: identification and compartmentalized expression of wnt-2, wnt-2b, and frizzled-4 mRNAs," Endocrinology, vol. 143, no. 7, pp. 2741-2749, 2002.

[22] D. Hanahan and R. A. Weinberg, "Hallmarks of cancer: the next generation," Cell, vol. 144, no. 5, pp. 646-674, 2011.

[23] A. Nowicki, J. Szenajch, G. Ostrowska et al., "Impaired tumor growth in colony-stimulating factor 1 (CSF-1)-deficient, macrophage-deficient op/op mouse: evidence for a role of CSF-1-dependent macrophages in formation of tumor stroma," International Journal of Cancer, vol. 65, no. 1, pp. 112-119, 1996.

[24] G. Pawelec, "Tumour escape: antitumour effectors too much of a good thing?" Cancer Immunology, Immunotherapy, vol. 53, no. 3, pp. 262-274, 2004.

[25] L. Yang and D. P. Carbone, "Tumor-host immune interactions and dendritic cell dysfunction," Advances in Cancer Research, vol. 92, pp. 13-27, 2004.

[26] L. M. Coussens and Z. Werb, "Inflammation and cancer," Nature, vol. 420, no. 6917, pp. 860-867, 2002.

[27] R. Kalluri and M. Zeisberg, "Fibroblasts in cancer," Nature Reviews Cancer, vol. 6, no. 5, pp. 392-401, 2006.

[28] S. Gillessen, Y. N. Naumov, E. E. S. Nieuwenhuis et al., "CD1drestricted $\mathrm{T}$ cells regulate dendritic cell function and antitumor 
immunity in a granulocyte-macrophage colony-stimulating factor-dependent fashion," Proceedings of the National Academy of Sciences of the United States of America, vol. 100, no. 15, pp. 8874-8879, 2003.

[29] T. A. Gonda, A. Varro, T. C. Wang, and B. Tycko, "Molecular biology of cancer-associated fibroblasts: can these cells be targeted in anti-cancer therapy?" Seminars in Cell and Developmental Biology, vol. 21, no. 1, pp. 2-10, 2010.

[30] J. A. Parrott, E. Nilsson, R. Mosher et al., "Stromal-epithelial interactions in the progression of ovarian cancer: influence and source of tumor stromal cells," Molecular and Cellular Endocrinology, vol. 175, no. 1-2, pp. 29-39, 2001. 


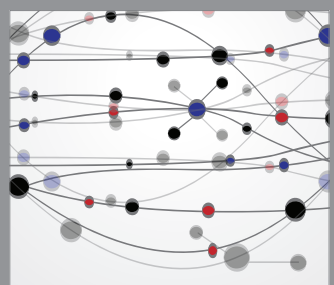

The Scientific World Journal
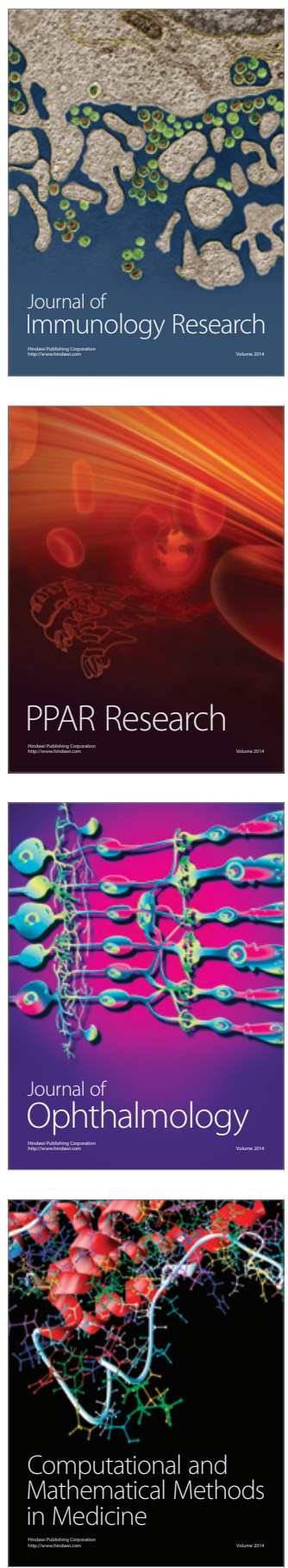

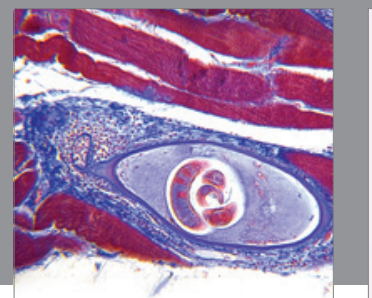

Gastroenterology

Research and Practice
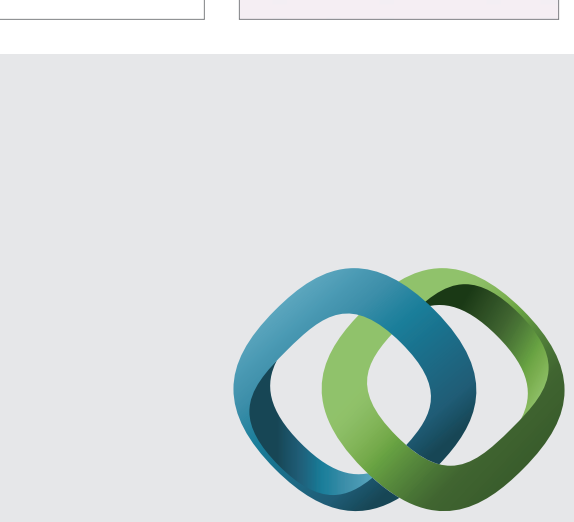

\section{Hindawi}

Submit your manuscripts at

http://www.hindawi.com
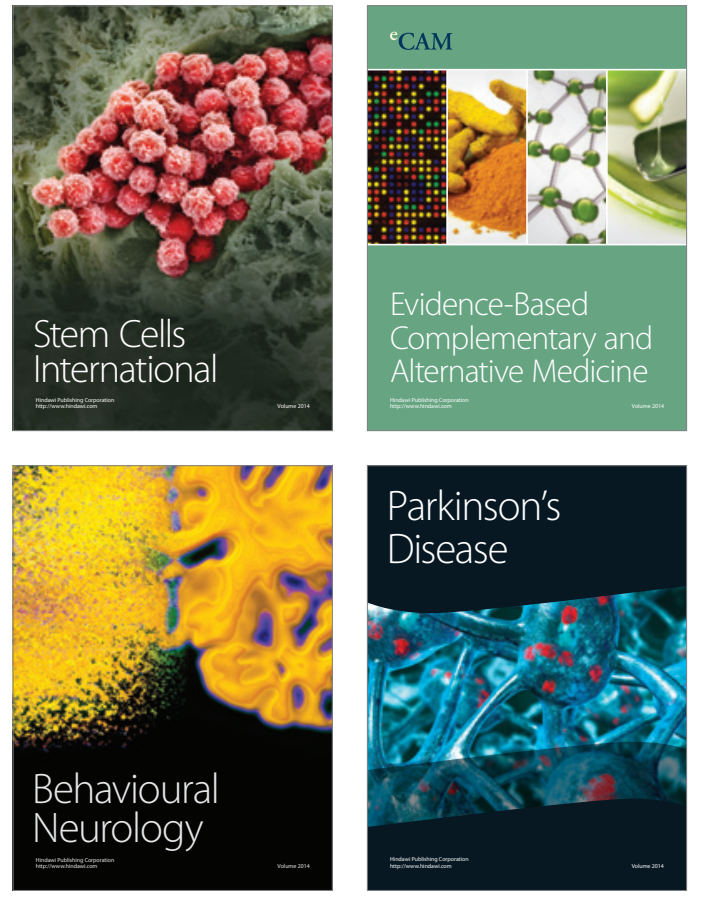
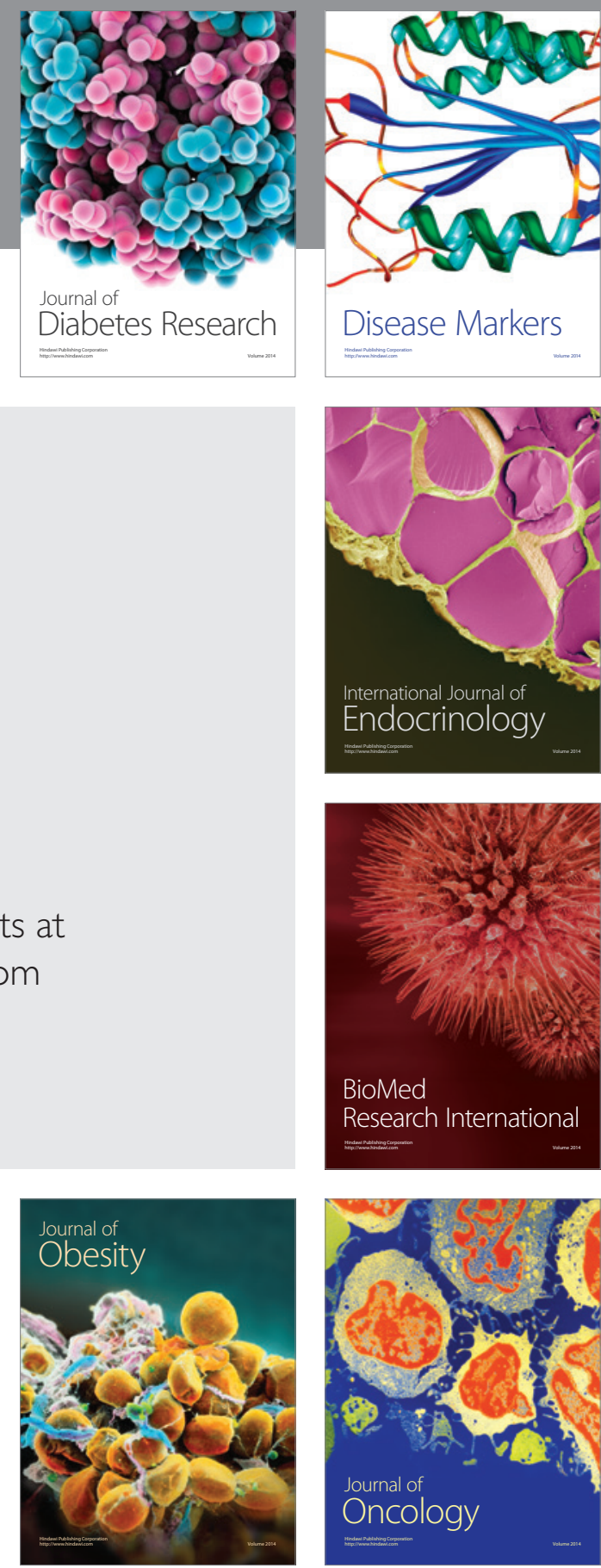

Disease Markers
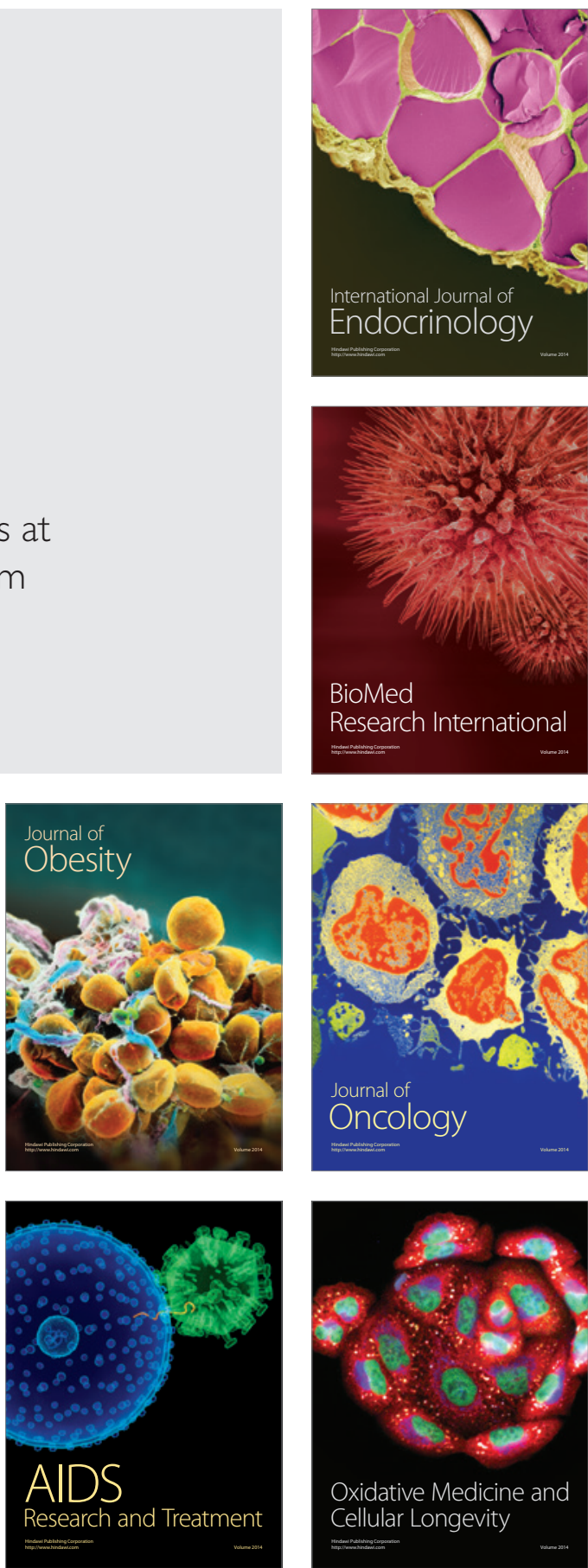